\title{
A Diagnostic Calculator for Detecting Glaucoma on the Basis of Retinal Nerve Fiber Layer, Optic Disc, and Retinal Ganglion Cell Analysis by Optical Coherence Tomography
}

\author{
José Manuel Larrosa, ${ }^{1}$ Javier Moreno-Montañés, ${ }^{2}$ José María Martinez-de-la-Casa,${ }^{3}$ Vicente Polo, ${ }^{1}$ \\ Álvaro Velázquez-Villoria, ${ }^{2}$ Clara Berrozpe, ${ }^{3}$ and Marta García-Granero ${ }^{4}$ \\ ${ }^{1}$ Ophthalmology Service, Hospital Universitario Miguel Servet, Faculty of Medicine, University of Saragossa, Saragossa, Spain \\ ${ }^{2}$ Department of Ophthalmology, Clínica Universidad de Navarra, Faculty of Medicine, Universidad of Navarra, Pamplona, Spain \\ ${ }^{3}$ Ophthalmology Service, Hospital Clínico San Carlos, Faculty of Medicine, Universidad Complutense de Madrid, Madrid, Spain \\ ${ }^{4}$ Unidad de Estadística, Department of Biochemistry and Genetics, University of Navarra, Pamplona, Spain
}

Correspondence: Javier MorenoMontañés, Department of Ophthalmology, Clínica Universidad de Navarra, Av. Pío XII, 36, Pamplona, Spain;

jmoreno@unav.es.

JML, JM-M, and JMM-d-1-C contributed equally to the work presented here and should therefore be regarded as equivalent authors.

Submitted: April 27, 2015

Accepted: August 1, 2015

Citation: Larrosa JM, Moreno-Montañés J, Martinez-de-la-Casa JM, et al. A diagnostic calculator for detecting glaucoma on the basis of retinal nerve fiber layer, optic disc, and retinal ganglion cell analysis by optical coherence tomography. Invest Ophthalmol Vis Sci. 2015;56:6788-6795. DOI:10.1167/iovs.15-17176
Purpose. The purpose of this study was to develop and validate a multivariate predictive model to detect glaucoma by using a combination of retinal nerve fiber layer (RNFL), retinal ganglion cell-inner plexiform (GCIPL), and optic disc parameters measured using spectraldomain optical coherence tomography (OCT).

Methods. Five hundred eyes from 500 participants and 187 eyes of another 187 participants were included in the study and validation groups, respectively. Patients with glaucoma were classified in five groups based on visual field damage. Sensitivity and specificity of all glaucoma OCT parameters were analyzed. Receiver operating characteristic curves (ROC) and areas under the ROC (AUC) were compared. Three predictive multivariate models (quantitative, qualitative, and combined) that used a combination of the best OCT parameters were constructed. A diagnostic calculator was created using the combined multivariate model.

REsults. The best AUC parameters were: inferior RNFL, average RNFL, vertical cup/disc ratio, minimal GCIPL, and inferior-temporal GCIPL. Comparisons among the parameters did not show that the GCIPL parameters were better than those of the RNFL in early and advanced glaucoma. The highest AUC was in the combined predictive model $(0.937 ; 95 \%$ confidence interval, 0.911-0.957) and was significantly $(P=0.0001)$ higher than the other isolated parameters considered in early and advanced glaucoma. The validation group displayed similar results to those of the study group.

Conclusions. Best GCIPL, RNFL, and optic disc parameters showed a similar ability to detect glaucoma. The combined predictive formula improved the glaucoma detection compared to the best isolated parameters evaluated. The diagnostic calculator obtained good classification from participants in both the study and validation groups.

Keywords: glaucoma diagnosis, OCT, optic disc, retinal ganglion cell, retinal nerve fiber layer
G laucoma is a frequent cause of blindness worldwide. ${ }^{1}$ The J modern standard for diagnosing glaucoma is based on analyses of structural and functional changes of the optic nerve head $(\mathrm{ONH})$. Visual field analysis is the functional study used most widely. Structural changes in the optic nerve are observed using different techniques. Optic disc photography conventionally has been used to evaluate the structural optic nerve damage in glaucoma; however, interobserver variability and moderate interobserver agreement suggest that fundus photography discriminates poorly between normal and glaucomatous optic nerves. ${ }^{2}$ Advances in ocular imaging techniques, principally the advent of optical coherence tomography (OCT), have facilitated assessment of the peripapillary retinal nerve fiber layer (RNFL). Different OCT methods have been introduced for quantitative and qualitative analyses of the RNFL, using glaucoma algorithms to measure the thickness along a 3.4mm-diameter circle centered on the optic disc. Recently, advances in OCT segmentation algorithms have facilitated the measurement of the thickness of the macular retinal ganglion cells-inner plexiform layers (GCIPL). ${ }^{3-5}$ Essentially, glaucoma causes retinal ganglion cell bodies and their axons to die. Current published studies have suggested that analysis of the macular GCIPL yields the same glaucoma diagnostic result as RNFL thickness. ${ }^{3-5}$

Recent articles have analyzed combinations of different structural parameters to improve the diagnostic ability to detect glaucoma; some analyzed the RNFL thickness and optic nerve parameters and others the peripapillary RNFL and macular GCIPL parameters. ${ }^{6-8}$ The purpose of the current study was to develop and validate multivariate predictive models to detect glaucoma by using a combination of optic nerve evaluation, RNFL thickness, and macular GCIPL parameters measured by Cirrus OCT (Carl Zeiss Meditec, Inc., Dublin, CA, USA) to improve the detection of glaucoma in the initial and advanced disease stages. 


\section{Materials AND Methods}

Patients with normal or glaucomatous eyes were recruited prospectively in three departments of ophthalmology: the Clínica Universidad de Navarra, Pamplona, Spain; Hospital Clínico S. Carlos, Universidad Complutense, Madrid, Spain; and Hospital Miguel Servet, Universidad of Saragossa, Saragossa, Spain. Institutional review boards/ethics committees approved the study. According to the committee, no written informed consent was needed for the glaucoma group because data were collected from regular clinical practice. All normal volunteers provided informed consent before entering the study, which adhered to the Declaration of Helsinki.

Complete ophthalmic examinations of all participants were performed, which included slit-lamp biomicroscopy, IOP measurement, dilated stereoscopic fundus examination, gonioscopy, and standard automated perimetry using the 24-2 Swedish interactive threshold algorithm (Humphrey field analyzer, Carl Zeiss Meditec, Inc.). All participants were Caucasian-Hispanic and had a spherical equivalent within 5.0 diopters (D) or less, astigmatism of $3.00 \mathrm{D}$ or less, bestcorrected visual acuity (VA) of 20/40 or better; no corneal or retinal pathology, amblyopia, or macular disease; no contraindication to pupillary dilation or intolerance to topical anesthetic or mydriatic agents; and no substantial media opacity.

The glaucoma group included patients with a clinical diagnosis of open-angle glaucoma identified by gonioscopy and visual field defects and an IOP that exceeded $21 \mathrm{~mm} \mathrm{Hg}$ on at least 3 different days. The visual field was classified as glaucomatous according to a glaucoma staging system (GSS), a modified version of the Hodapp-Parrish-Anderson classification. ${ }^{9}$ This classification allows for stage assignment based on visual field damage. Stage 0 indicates ocular hypertension, stage 1 early glaucoma, stage 2 moderate glaucoma, stage 3 advanced glaucoma, stage 4 severe glaucoma, and stage 5 blindness. Visual field damage was reproducible in at least three reliable and consecutive visual field tests performed on different days. In patients with bilateral glaucoma, only a randomized eye was included. The normal group included participants with an IOP of $21 \mathrm{~mm} \mathrm{Hg}$ or lower, normal visual fields, and no familial history of glaucoma. The normal group included participants without ocular diseases consecutively recruited from hospital staff members, nurses, relatives of patients, and patients referred for a routine VA examination.

\section{OCT Acquisition and Analysis}

After pupillary dilation with $1 \%$ tropicamide, an OCT evaluation using Cirrus OCT was performed on the same day as visual field analysis. Before the study, the manufacturer calibrated the OCTs at the three hospitals. The experienced operators who performed the OCT examinations differed from the examiner who performed visual field testing and was masked to the other findings.

Three OCT volume scans $(200 \times 200$ axial scans $)$ centered on the optic disc were obtained for each eye. Retinal nerve fiber layer data were evaluated automatically using the Cirrus OCT system software version 6.0. After optic discs were scanned, three new scans $(200 \times 200$ axial scans $)$ were obtained in the macular cube to analyze GCIPLs. The OCT signal strength also was analyzed. Only the best signal strength scan from the three obtained scans was selected for the RNFL and GCIPLs analysis.

The optic nerve cube evaluated the RNFL thickness and optic disc parameters. Retinal nerve fiber layer parameters evaluated were the global RNFL average (micrometers) and RNFL thickness in the superior, inferior, nasal, and temporal quadrants. The 12-hour clock positions were not included. The optic disc parameters were the rim area $\left(\mathrm{mm}^{2}\right)$, average cup/ disc $(\mathrm{C} / \mathrm{D})$ ratio, vertical C/D ratio, and cup volume $\left(\mathrm{mm}^{3}\right)$.

The macular cube in Cirrus OCT combined the thicknesses of the GCIPL layers and the inner plexiform layer, because the Cirrus software cannot distinguish between the two, and the combined thickness indicates the health of the GCIPLs. The GCIPL data analyzed from the macular cube were the average, minimal (lowest GCIPL thickness over a single meridian crossing the annulus), and sectoral (superotemporal, superior, superonasal, inferonasal, inferior, inferotemporal) thicknesses measured in an elliptical annulus around the fovea. The vertical dimension was 0.5 to $2 \mathrm{~mm}$ from the fovea, and the horizontal dimension was 0.6 to $2.4 \mathrm{~mm}$ from the fovea.

Cirrus software automatically organized all RNFL, optic disc parameters, and GCIPL values into three groups: within normal limits (green), borderline (yellow), and out of normal limits (red). Only cases with a signal strength of 6 or higher were analyzed. Cases with signal strength of 5 or lower were rejected.

\section{Data Study Analysis}

Five hundred eyes (248 right, 252 left eyes) of 500 participants ( 251 men, 249 women) were enrolled. The mean \pm standard deviation (SD) age was $68.3 \pm 8.7$ years. One hundred fifty-one eyes were normal, 124 eyes had ocular hypertension (grade 0 according to the GSS classification), and 215 eyes were glaucomatous. The mean $\pm \mathrm{SD}$ age in the normal group was $67.7 \pm 7.7$ years and $69.2 \pm 9.9$ years in the glaucomatous group $(P=0.065)$.

\section{Data Validation Analysis}

One hundred eighty-seven eyes (93 right, 94 left eyes) of 187 participants (91 men, 96 women) were included in this group. This validation group was created at the same time as the study group so that 500 eyes were chosen for the analysis group and the other eyes for the validation group. Eyes were distributed randomly in both groups. Fifty-seven eyes were normal, 48 eyes were hypertensive, and 82 were glaucomatous. The mean \pm SD age in the normal group was $67.0 \pm 9.2$ years and $69.7 \pm$ 11.4 years in the glaucomatous group $(P=0.072)$. Demographic data from the study and validation groups are shown in Table 1. No statistical differences were found between the groups in any evaluated parameters.

\section{Statistical Analysis}

The Shapiro-Wilk test was used to assess the normality of the quantitative variables. Normally distributed variables were summarized using means $\pm \mathrm{SD}$, and nonnormally distributed variables were summarized using median and interquartile ranges (25th and 75 th percentiles). To compare quantitative variables between two independent groups (sex, normal/ glaucomatous group), the Mann-Whitney $U$ test was used for nonnormally distributed variables (all but age), and the twosample Student's $t$-test was used for normally distributed variables (age only). The $\chi^{2}$ test was used to compare qualitative variables between independent groups.

Three different predictive models were evaluated using multivariate logistic regression: one from the quantitative data from the RNFL, optic disc, and GCIPLs (model 1); another using qualitative data (green, yellow, and red [model 2]), and combined qualitative and quantitative data (model 3). These models were analyzed in the study and validation groups. The receiver operating characteristic curves (ROC) were used to determine the discriminatory capabilities between healthy and 
Table 1. Demographic Data From the Study and Validation Groups

\begin{tabular}{lccc}
\hline \multicolumn{1}{c}{ Data } & Study Group & Validation Group & $P$ Value \\
\hline No. of cases & 500 & 187 & 0.83 \\
Mean \pm SD age, y & $68.3 \pm 8.7$ & $88.2 \pm 10.4$ & 0.12 \\
Mean \pm SD visual field index & $80.0 \pm 32.1$ & $96(51.3)$ & 0.93 \\
No. of women (\%) & $249(49.8)$ & $93(49.7)$ & 0.97 \\
No. of right eyes (\%) & $248(49.6)$ & $82(43.9)$ & \\
No. of glaucomatous eyes (\%) & $215(43)$ & $37(45.1)$ & 0.95 \\
GSS (\%) & & $21(25.6)$ \\
Stage 1 & $92(42.7)$ & $16(19.5)$ \\
Stage 2 & $71(33)$ & $7(8.5)$ \\
Stage 3 & $36(16.7)$ & $1(1.2)$ \\
Stage 4 & $16(7.4)$ & 0.53 \\
Stage 5 & 0 & \\
\hline
\end{tabular}

GSS, glaucoma staging system.

glaucomatous eyes. The best parameters from the RNFL, optic disc, and GCIPL analyses were compared to the multivariate models in early moderate and advanced glaucoma. AUCs were compared using the Hanley-McNeil method for paired data. ${ }^{10}$ Sensitivities of $85 \%$ and $95 \%$ fixed specificities were calculated.

Bootstrapping also was used to internally validate the regression model and was implemented by constructing a number of resamples $(k=100)$ of the dataset (of equal size) obtained by random sampling with replacement from the original dataset. ${ }^{11}$ Bootstrapping allows use of a complete dataset for model development, evaluation, and validation and currently is considered the resampling method of choice. ${ }^{12}$ Data were evaluated using SPSS version 20.0.1 software (SPSS, Inc., Chicago, IL, USA), STATA version 12.0 software (Stata Corp, College Station, TX, USA), and MedCalc version 11.2 (MedCalc Software, Mariakerke, Belgium).

\section{Results}

\section{Areas Under the ROC Curves}

Table 2 shows AUC values, best sensitivity and specificity values, and sensitivity for the $85 \%$ and $95 \%$ fixed specificities from the RNFL and optic disc values from the study group. The best AUCs were the RNFL inferior quadrant (AUC, 0.867; 95\% confidence interval [CI], 0.835-0.896), and the average RNFL (AUC, 0.849; 95\% CI, 0.815-0.880); however, the RNFL inferior quadrant sensitivity is higher for the $85 \%$ and $95 \%$ fixed specificities than the average RNFL. The best AUC of the optic disc was the vertical C/D ratio (AUC, $0.820 ; 95 \% \mathrm{CI}, 0.783-0.863$ ) with values similar to the rim area.

Areas under the curve of the GCIPL values in the study group are shown in Table 3. The best AUCs were the minimal value GCIPL (AUC, $0.868 ; 95 \% \mathrm{CI}, 0.835-0.896$ ), the inferior temporal quadrant GCIPL (AUC, 0.867; 95\% CI, 0.834-0.896), and the average GCIPL (AUC, 0.837; 95\% CI, 0.801-0.868). Comparison of the RNFL inferior quadrant and average RNFL with the minimal value GCIPL and inferior quadrant GCIPL did not show significant differences, suggesting that GCIPL analysis does not improve the ability to diagnose glaucoma based on the RNFL thickness.

In the study group, glaucoma was classified according to the GSS system. Early moderate glaucoma was defined as grades 1 and 2 and advanced glaucoma as grades 3, 4, and 5 . Comparisons between the AUCs of the best RNFL thickness and those of the best GCIPL thicknesses did not identify significant differences in early moderate and advanced glaucoma (Table 4).

\section{New Multivariate Predictive Models}

The best predictive parameters of the RNFL, optic disc, and GCIPLs were selected based on multivariate regression analysis, and three models were obtained. Model 1 included quantitative data, model 2 qualitative data, and model 3

TABLe 2. Area Under the ROCs, Best Sensitivity-Specificity Balance, Sensitivity for $85 \%$ and $95 \%$ Fixed Specificities of the RNFL and Optic Disc Values in the Study Group (RNFL and Optic Disc Parameters)

\begin{tabular}{|c|c|c|c|c|c|}
\hline \multirow[b]{2}{*}{ Site } & \multirow[b]{2}{*}{ AUC (95\% CI) } & \multirow[b]{2}{*}{$P$ Value } & \multirow[b]{2}{*}{ Sensitivity $\% /$ Specificity $\%$} & \multicolumn{2}{|c|}{ Sensitivity by Specificity, \% } \\
\hline & & & & 85\% Specificity & 95\% Specificity \\
\hline \multicolumn{6}{|l|}{ RNFL } \\
\hline Average & $0.849(0.815-0.880)$ & 0.0001 & $80.4 / 78.9$ & 71.0 & 50.5 \\
\hline Superior & $0.814(0.778-0.848)$ & 0.0001 & $69.3 / 83.7$ & 65.6 & 49.4 \\
\hline Inferior & $0.867(0.835-0.896)$ & 0.0001 & $80.5 / 80.7$ & 74.4 & 56.3 \\
\hline Temporal & $0.681(0.639-0.722)$ & 0.0001 & $52.6 / 82.5$ & 48.8 & 25.6 \\
\hline Nasal & $0.646(0.602-0.688)$ & 0.0001 & $62.8 / 62.8$ & 33.0 & 14.4 \\
\hline \multicolumn{6}{|l|}{ Optic disc } \\
\hline Rim area & $0.819(0.791-0.861)$ & 0.0001 & $67.4 / 85.9$ & 67.9 & 54.0 \\
\hline Average $\mathrm{C} / \mathrm{D}$ ratio & $0.787(0.749-0.822)$ & 0.0001 & $64.6 / 85.9$ & 63.7 & 40.0 \\
\hline Vertical C/D ratio & $0.820(0.783-0.863)$ & 0.0001 & $64.2 / 89.1$ & 67.4 & 54.9 \\
\hline Cup volume & $0.708(0.666-0.748)$ & 0.0001 & $72.5 / 63.8$ & 41.9 & 17.7 \\
\hline
\end{tabular}


TABLe 3. Area Under the ROCs, Best Sensitivity-Specificity Balance, Sensitivity for $85 \%$ and $95 \%$ Fixed Specificities of GCIPL Values in the Study Group (Ganglion Cell Thickness)

Sensitivity by Specificity, \%

\begin{tabular}{|c|c|c|c|c|c|}
\hline Site & AUC $(95 \%$ CI $)$ & $P$ Value & Sensitivity $\% /$ Specificity $\%$ & $85 \%$ Specificity & $95 \%$ Specificity \\
\hline Average & $0.837(0.801-0.868)$ & 0.0001 & $81.8 / 74.3$ & 66.1 & 40.9 \\
\hline Superior & $0.795(0.757-0.829)$ & 0.0001 & $69.7 / 76.4$ & 57.6 & 34.4 \\
\hline Inferior & $0.836(0.802-0.869)$ & 0.0001 & $67.9 / 89.1$ & 71.1 & 48.3 \\
\hline Superotemporal & $0.814(0.777-0.847)$ & 0.0001 & $66.5 / 84.8$ & 64.5 & 33.0 \\
\hline Superonasal & $0.747(0.706-0.784)$ & 0.0001 & $72.1 / 67.6$ & 46.1 & 29.8 \\
\hline Inferotemporal & $0.867(0.834-0.896)$ & 0.0001 & $74.5 / 85.9$ & 73.2 & 59.1 \\
\hline Inferonasal & $0.796(0.758-0.831)$ & 0.0001 & $74.4 / 72.2$ & 56.3 & 42.8 \\
\hline Minimum & $0.868(0.835-0.896)$ & 0.0001 & $88.4 / 72.2$ & 73.4 & 44.7 \\
\hline
\end{tabular}

combined qualitative and quantitative data. Table 5 and Figure 1A show the AUCs of the three models. Model 3 obtained the best AUC. We exported data to Stata 12 and compared models using Fitstat software. The output shows that the combined model 3 was considered the simplest, according to the Bayesian information criterion (BIC). Because of space limitations, only the model 3 formula is shown here:

Model 3 predicted probability $=$

$$
\begin{aligned}
e^{\wedge} & (0.905+0.044 \times \text { age }-1.477 \times(\mathrm{SNGCC}=\text { yellow }) \\
& -1.190 \times(\mathrm{SNGCC}=\text { red })+1.403 \times(\mathrm{STGCC}=\text { yellow }) \\
& +1.095 \times(\mathrm{STGCC}=\text { red })+1.455 \times(\mathrm{MCGC}=\text { yellow }) \\
& +1.109 \times(\mathrm{MCGC}=\text { red })+0.006 \times(\mathrm{CDAC}=\text { yellow }) \\
& +2.231 \times(\mathrm{CDAC}=\text { red })+0.583 \times(\mathrm{CDAC}=\text { gray }) \\
& -0.034 \times \mathrm{ITGC}-0.035 \times \mathrm{IRNFL}-0.099 \times \mathrm{CDA}(\times 100) \\
& +0.117 \times \mathrm{VCD}(\times 100)) /\left(1+e^{\wedge}(0.905+0.044 \times \text { age }\right. \\
& -1.477 \times(\mathrm{SNGCC}=\text { yellow })-1.190 \times(\mathrm{SNGCC}=\mathrm{red}) \\
& +1.403 \times(\mathrm{STGCC}=\text { yellow })+1.095 \times(\mathrm{STGCC}=\mathrm{red}) \\
& +1.455 \times(\mathrm{MCGC}=\text { yellow })+1.109 \times(\mathrm{MCGC}=\text { red }) \\
& +0.006 \times(\mathrm{CDAC}=\text { yellow })+2.231 \times(\mathrm{CDAC}=\mathrm{red}) \\
& +0.583 \times(\mathrm{CDAC}=\text { gray })-0.034 \times \mathrm{ITGC}-0.035 \\
& \times \mathrm{IRNFL}-0.099 \mathrm{CDA}(\times 100)+0.117 \times \mathrm{VCD}(\times 100)))
\end{aligned}
$$

\begin{tabular}{|c|c|c|c|c|c|}
\hline \multirow[b]{2}{*}{ Site } & \multirow[b]{2}{*}{ AUC (95\% CI) } & \multirow[b]{2}{*}{$P$ Value } & \multirow[b]{2}{*}{ Sensitivity $\% /$ Specificity $\%$} & \multicolumn{2}{|c|}{ Sensitivity by Specificity, $\%$} \\
\hline & & & & $85 \%$ Specificity & 95\% Specificity \\
\hline \multicolumn{6}{|l|}{ Early-moderate glaucoma } \\
\hline Average RNFL & $0.871(0.828-0.907)$ & 0.0001 & $77.3 / 87.1$ & 77.9 & 53.5 \\
\hline Inferior RNFL & $0.877(0.834-0.921)$ & 0.0001 & $77.3 / 86.4$ & 77.7 & 59.8 \\
\hline Vertical $\mathrm{C} / \mathrm{D}$ ratio & $0.843(0.796-0.882)$ & 0.0001 & $73.6 / 84.8$ & 72.3 & 53.6 \\
\hline Minimal GCIPL & $0.868(0.824-0.905)$ & 0.0001 & $86.5 / 77.3$ & 73.6 & 39.7 \\
\hline Infero-temporal GCIPL & $0.869(0.825-0.905)$ & 0.0001 & $69.4 / 92.4$ & 76.1 & 60.1 \\
\hline \multicolumn{6}{|l|}{ Advanced glaucoma } \\
\hline Average RNFL & $0.936(0.891-0.967)$ & 0.0001 & $86.3 / 91.7$ & 91.7 & 76.5 \\
\hline Inferior RNFL & $0.933(0.887-0.964)$ & 0.0001 & $88.5 / 92.4$ & 93.2 & 84.2 \\
\hline Vertical $\mathrm{C} / \mathrm{D}$ ratio & $0.911(0.860-0.948)$ & 0.0001 & $84.6 / 90.9$ & 88.6 & 78.9 \\
\hline Minimal GCIPL & $0.928(0.881-0.961)$ & 0.0001 & $86.5 / 93.2$ & 88.4 & 63.9 \\
\hline Infero-temporal GCIPL & $0.953(0.911-0.978)$ & 0.0001 & $86.6 / 94.2$ & 92.3 & 86.5 \\
\hline
\end{tabular}

TABLE 4. Area Under the ROCs, Best Sensitivity-Specificity Balance, Sensitivity for $85 \%$ and $95 \%$ Fixed Specificities of RNFL and GCIPL Values in Early and Advanced Glaucomas (Study Group) superotemporal GCIPL color; MCGC is the minimal GCIPL color; CDAC C/D ratio is average color; ITGC is the inferotemporal GCIPL value; IRNFL is the inferior quadrant RNFL value; CDA C/D is the average value; and VCD is the vertical $\mathrm{C} / \mathrm{D}$ value.

We suggest the following cutoff points: predicted probability of $<0.3$ is the low probability; 0.3 to 0.6 is the intermediate probability range; and $>0.6$ is high probability. In the study group, only $9.3 \%$ of eyes with a low score were glaucomatous, $41 \%$ of eyes with an intermediate score were glaucomatous, and finally, $91 \%$ of the high-score group were glaucomatous. In the validation group, the percentages were 9.3\%, 52\%, and $91 \%$, respectively. Therefore, the proposed cutoffs points seem adequate.

Table 6 shows the odds ratios (ORs) of the best parameters of the GCIPLs, optic disc, and RNFL obtained in the combined multivariate linear regression model. The ROC from model 3 and best parameters of the RNFL, optic disc, and GCIPL analysis in the study group are shown in Figure 1. AUCs of model 3 were significantly $(P<0.001)$ higher than all the best parameters evaluated (Table 7 ), suggesting that the ability to detect glaucoma using the multivariate model is better than the best parameters of the RNFL, optic disc, and GCIPL analysis.

Figure 2 shows the ROC curve in patients with early moderate and advanced glaucoma. In early glaucoma, model 3 obtained significantly higher AUCs than all isolated parameters evaluated $(P<0.001)$. However, in advanced glaucoma, model 3 AUCs were only higher than three parameters: the minimal 
TABLE 5. Area Under the ROCs, Best Sensitivity-Specificity Balance, Sensitivity for $85 \%$ and $95 \%$ Fixed Specificities of New Predictive Multivariate Model

Sensitivity by Specificity, \%

\begin{tabular}{|c|c|c|c|c|c|}
\hline Model & AUC $(95 \%$ CI $)$ & $P$ Value & Sensitivity $\% /$ Specificity $\%$ & $85 \%$ Specificity & 95\% Specificity \\
\hline \multicolumn{6}{|l|}{ Study group } \\
\hline Model 1 & $0.912(0.883-0.935)$ & 0.0001 & $85.1 / 84.5$ & 84.2 & 68.4 \\
\hline Model 2 & $0.926(0.898-0.948)$ & 0.0001 & $91.3 / 83.7$ & 89.3 & 67.4 \\
\hline Model $3^{*}$ & $0.937(0.911-0.957)$ & 0.0001 & $83.8 / 91.2$ & 87.9 & 77.8 \\
\hline \multicolumn{6}{|c|}{ Validation group } \\
\hline Model 1 & $0.926(0.879-0.959)$ & 0.0001 & $81.7 / 95.2$ & 86.6 & 81.7 \\
\hline Model 2 & $0.937(0.892-0.967)$ & 0.0001 & $82.9 / 96.1$ & 86.6 & 82.9 \\
\hline Model 3 & $0.932(0.886-0.963)$ & 0.0001 & $84.1 / 93.3$ & 87.8 & 78.1 \\
\hline
\end{tabular}

* Differences between models 1 and 3 in $\mathrm{AUC}=0.0268$ (95 CI, 0.0100-0.0436; $P=0.0017$ ).

GCIPL $(P=0.01)$, inferior temporal GCIPL $(P=0.04)$, and average RNFL $(P=0.04)$.

\section{Validation Analysis}

The three regression models were analyzed in the validation group and the calculated AUCs were similar to those obtained for the study group; in all models, the AUCs were higher than 0.90 (Table 5). To facilitate the external validation of model 3 , we include the Website from which to download the Excel (Microsoft, Redmond, WA, USA) formula with the diagnostic categories (http://www.oftared.com/docs/Glaucoma\% 20Diagnostic\%20Calculator.xlsx, in the public domain) (Fig. 3).

A

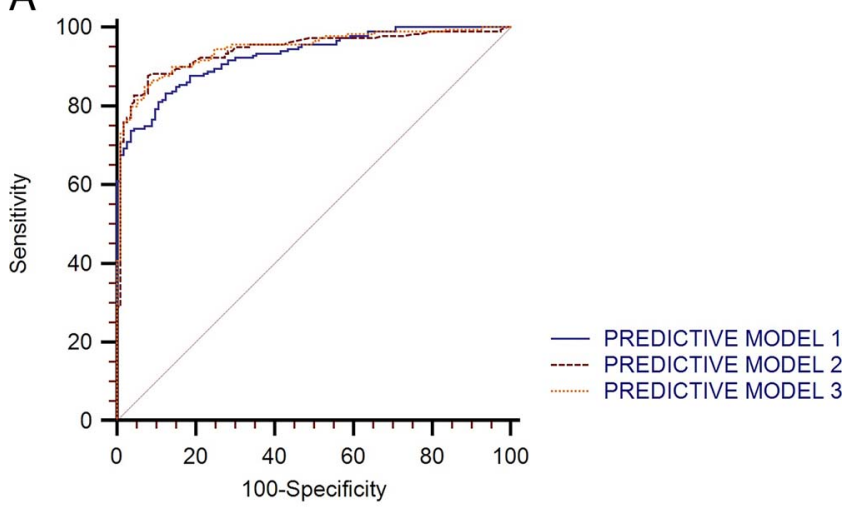

B

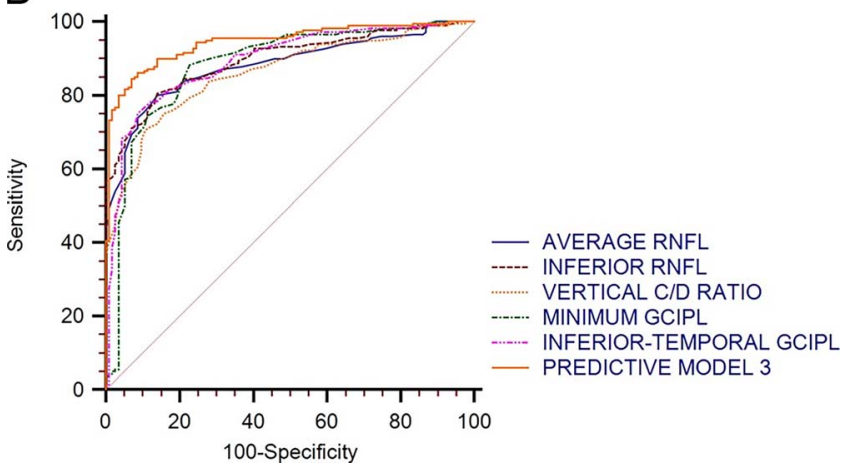

Figure 1. (A) The ROC from the three predictive models. (B) The ROC from the best parameters of the RNFL, optic disc, and GCIPLs and the new parameter (model 3 ) in all eyes.
The bootstrap method was the internal validation method, and its estimates agreed closely with the parameters obtained by regression analysis of the original dataset. Furthermore, the bootstrap estimate of the AUC from model 1 was 0.918 (95\% CI, 0.891-0.943), 0.934 (95\% CI, 0.909-0.957) from model 2, and 0.922 (95\% CI, 0.917-0.938) from model 3.

\section{Discussion}

Advances in OCT technology have enabled more detailed and precise quantitative assessment of glaucomatous structural changes, through circumpapillary RNFL thickness measurements and, more recently, GCIPL thickness measurements. Considering the current results, GCIPL assessment does not improve the ability to diagnose glaucoma from the peripapillary RNFL evaluation. These results generally agreed with previous reports. ${ }^{13-15}$ However, RNFL and GCIPL assessments target different neuroretinal areas, and they may be potentially complementary to overcome the incidence of false positive results of some OCT RNFL parameters. ${ }^{16}$ Thus, in Cirrus OCT, Leal-Fonseca et al. ${ }^{16}$ reported an $11 \%$ false positive rate in the average RNFL color code and $13 \%$ in the superior and inferior quadrants and average $\mathrm{C} / \mathrm{D}$ ratio. This supports the combined use of parameters from different retinal areas for diagnosing glaucoma. Consequently, multivariate models predictive of glaucoma have been proposed to improve the diagnostic ability of these OCT parameters that yield results that are highly correlated and somewhat redundant. Previous studies have used learning classifiers, linear discriminant functions, principal component analysis, and logistic regression analy$\operatorname{ses}^{6-8}$ to improve the OCT diagnostic value. Some studies combined ONH and RNFL parameters that evaluated the status of GC axons at different locations, ${ }^{17}$ whereas other studies combined ONH, RNFL, and macular GCIPL complex parameters, which accumulate information about different retinal anatomic areas. ${ }^{6-8}$ However, those studies did not show the mathematical functions in their reports or provide a Web link to allow external validation or simple implementation of the proposed function in a given patient. It is important that such information be accessible; otherwise, the value of the function is limited to the authors and cannot be compared.

We performed an internal validation of the formula, which ensured that the proposed model was robust and guaranteed that the function would be useful in future datasets. In addition, compared to previous reports, ${ }^{6-8,17}$ we presented our diagnostic calculator for external validation directly and through a Web link. We also tested and validated the functions in larger populations compared with comparable reports such as that of Mwanza et al. ${ }^{8}$ (687 subjects compared to 253 
Table 6. Odds Ratio (95\% CI) of the Best Parameters of the GCIPL, Optic Disc Parameters, and RNFL Values Obtained by Combined Multivariate Logistic Regression (Model 3)

\begin{tabular}{|c|c|c|c|c|c|c|c|}
\hline \multirow[b]{2}{*}{ Characteristic } & \multirow[b]{2}{*}{ Beta } & \multirow[b]{2}{*}{ SE (B) } & \multirow[b]{2}{*}{ Wald Test } & \multirow[b]{2}{*}{$P$ Value } & \multirow[b]{2}{*}{ OR } & \multicolumn{2}{|c|}{$95 \% \mathrm{CI}$} \\
\hline & & & & & & Lower & Upper \\
\hline Age, y & 0.044 & 0.015 & 8.647 & 0.003 & 1.045 & 1.015 & 1.076 \\
\hline Superonasal GCIPL color (vs. green) & & & 6.483 & 0.039 & & & \\
\hline Yellow & -1.477 & 0.611 & 5.842 & 0.016 & 0.228 & 0.069 & 0.756 \\
\hline Red & -1.190 & 0.745 & 2.547 & 0.111 & 0.304 & 0.071 & 1.312 \\
\hline Superotemporal GCIPL color (vs. green) & & & 7.114 & 0.029 & & & \\
\hline Yellow & 1.403 & 0.568 & 6.102 & 0.014 & 4.067 & 1.336 & 12.378 \\
\hline Red & 1.095 & 0.642 & 2.910 & 0.088 & 2.990 & 0.850 & 10.523 \\
\hline Minimal GCIPL color (vs. green) & & & 8.216 & 0.016 & & & \\
\hline Yellow & 1.455 & 0.554 & 6.906 & 0.009 & 4.285 & 1.448 & 12.686 \\
\hline Red & 1.109 & 0.556 & 3.985 & 0.046 & 3.033 & 1.020 & 9.012 \\
\hline Cup/disc ratio average color (vs. green) & & & 16.593 & 0.001 & & & \\
\hline Yellow & 0.006 & 0.494 & $1.3 \mathrm{E}-04$ & 0.991 & 1.006 & 0.382 & 2.649 \\
\hline Red & 2.231 & 0.565 & 15.563 & $<0.001$ & 9.305 & 3.072 & 28.184 \\
\hline Gray & 0.583 & 0.523 & 1.242 & 0.265 & 1.791 & 0.643 & 4.992 \\
\hline Inferotemporal GCIPL value & -0.034 & 0.017 & 3.913 & 0.048 & 0.967 & 0.935 & 1.000 \\
\hline Inferior RNFL thickness & -0.035 & 0.008 & 19.459 & $<0.001$ & 0.966 & 0.951 & 0.981 \\
\hline Average $\mathrm{C} / \mathrm{D}$ ratio, value $\times 100$ & -9.864 & 3.274 & 9.076 & 0.003 & 0.906 & 0.869 & 0.944 \\
\hline Vertical C/D ratio, value $\times 100$ & 11.718 & 3.312 & 12.519 & $<0.001$ & 1.124 & 1.078 & 1.172 \\
\hline Constant & 0.905 & 1.861 & 0.237 & 0.627 & & & \\
\hline
\end{tabular}

$P$ values in bold are significant. OR, odds ratio; SE (B), standard error of beta.

subjects). Furthermore, our subjects were recruited from three hospitals and were representative of the Spanish population. Finally, the availability of our function allows it to be compared in larger populations and with different ethnic and sociogeographic features.

The function significantly improved the diagnostic value of the single OCT parameters (Table 7). It is interesting that the RNFL parameters, such as the average RNFL or inferior RNFL, had AUCs (0.849 and 0.867 , respectively) similar to the best GCIPL parameters, such as the minimal and inferior-temporal GCIPLs (AUCs, 0.868 and 0.867 , respectively), whereas the ONH parameters such as the vertical C/D ratio had smaller AUC \& areas. However, the factor with the highest OR in the The $\mathrm{C} / \mathrm{D}$ ratio adds information to the model about an anatomical feature that does not overlap with other measurements of RNFL or GCIPL parameters. In contrast, the RNFL and GCIPL parameters assess different aspects of the RNFL thickness that are correlated strongly between them. The multivariate model includes the most relevant factors; in the current study, the GCIPL parameters had more weight in the formula (better odds ratios [ORs]) than the RNFL parameters

Table 7. Differences in the AUC Among the Best Quantitative Parameters From the RNFL or From the GCIPL and Combined Predictive Parameter (Model 3) in the Study Group

\begin{tabular}{lccc}
\hline \multicolumn{1}{c}{ Model Comparison } & $\begin{array}{c}\text { Differences } \\
\text { Between AUCs }\end{array}$ & $\begin{array}{c}\boldsymbol{P} \% \text { CI } \\
\text { Value }\end{array}$ \\
\hline $\begin{array}{l}\text { Model } 3 \text { vs. average RNFL } \\
\text { Model 3 vs. inferior }\end{array}$ & 0.0818 & $0.0551-0.108<0.0001$ \\
$\quad$ quadrant RNFL & 0.0641 & $0.0384-0.0899<0.0001$ \\
$\begin{array}{l}\text { Model 3 vs. minimal GCIPL } \\
\text { Model 3 vs. inferotemporal }\end{array}$ & 0.0688 & $0.0416-0.0961<0.0001$ \\
$\quad \begin{array}{llll}\text { GCIPL } \\
\text { Model 3 vs. vertical C/D ratio }\end{array}$ & 0.0517 & $0.0233-0.0802<0.0001$ \\
\hline
\end{tabular}

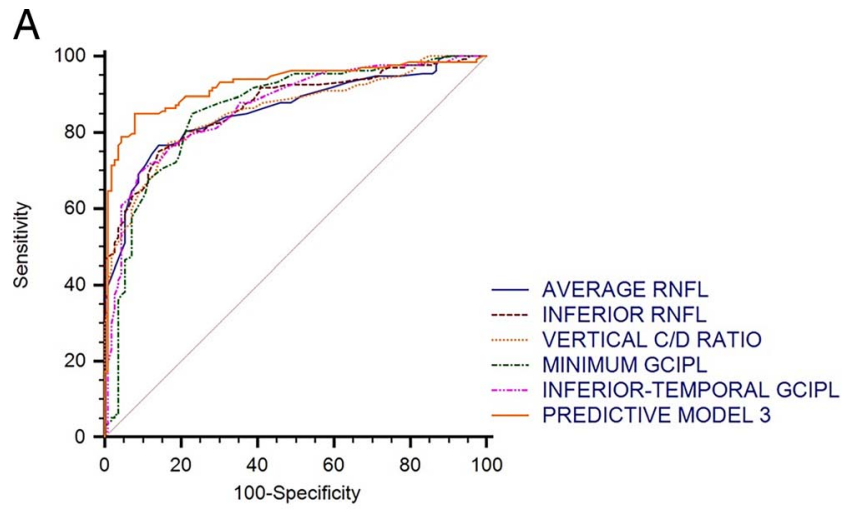

B

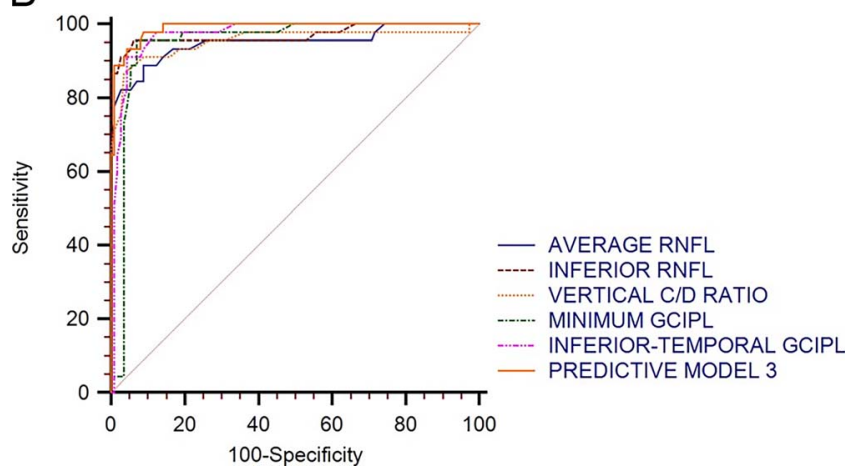

Figure 2. (A) The ROC from the best parameters of the RNFL and GCIPLs and a new parameter (model 3) in early moderate glaucoma. (B) The ROC from the best parameters of the RNFL and GCIPLs and a new parameter (model 3 ) in advanced glaucoma. 
GLAUCOMA DIAGNOSTIC CALCULATOR

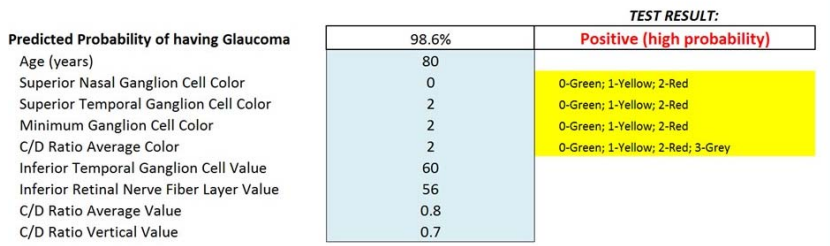

Figure 3. Printout of an Excel file from one case combining the nine parameters of the glaucoma diagnostic calculator (blue). The automatic positive result indicates a high probability of glaucoma.

(Table 6). Therefore, GCIPL assessment showed that the peripapillary RNFL thickness evaluation was more advantageous for diagnosing glaucoma. The low RNFL ORs in the formula suggested that the information about these parameters do not add much to the GCIPL measurements and supported the idea that the RNFL and GCIPL data mainly overlap.

Performing OCT often depends on the disease severity. It is easier to distinguish advanced glaucoma stages, but the function should not be restricted to detection in patients with early or moderate glaucoma. This approach avoids restricting the range in the test measures and therefore attenuating correlations among variables that can result in falsely low estimates of factor loading. The current function was not limited to early damage but covers all disease stages, unlike other reports. ${ }^{8}$ Compared to reports that included patients with all degrees of glaucoma severity, ${ }^{17}$ we also provided the diagnostic performance in the early-moderate and advanced stages. The diagnostic ability of the proposed multivariate function outperforms the diagnostic value of single parameters in early moderate and advanced glaucoma (Figs. 1, 2).

Models 2 and 3 showed similar levels of performance in the study series, and it is true that quantitative outcomes are usually preferred to categorizations, suggesting that perhaps the simpler model should be preferred. However, in this case, we must bear in mind that the qualitative measurements are not simply cutoff values of the original quantitative ones but are attributes provided by the Cirrus OCT (as indicated by the manufacturer), which take into account more patient information (like age and optic disc size) to assign a result to a given category. Thus, the information provided by the quantitative measurement is enriched (like the "gray" category, which indicates that the optic disc size is unusually small or big). Therefore, model 3 was finally selected for the Excel calculator.

The current study had limitations. All subjects were from the same geographic region despite the large number of participants and involvement of three hospitals from different cities. However, the availability of our formula through a Web link allows future testing of the function in different geographic and ethnic settings, similar to other OCT normative databases before being included in any software. The diagnostic color codes are based on the commercial version of the Cirrus OCT currently available. Their normative databases may be updated and modified in the future, and the results should not be extrapolated directly to other OCT devices. Another limitation of the proposed function was that subjects with macular disease, such as age-related macular degeneration (AMD), were excluded. Age-related macular degeneration is a relevant condition that may be considered in glaucoma suspects. The important weight of the GCIPL parameters in the formula should be considered and, therefore, implementation of this function in the presence of any macular condition is not strongly recommended. Despite these limitations, this diagnostic calculator is easy to use (Fig. 3) and includes only 9 parameters and the optic disc size as a gray color code, indicating a size not evaluated by Cirrus OCT. The
A
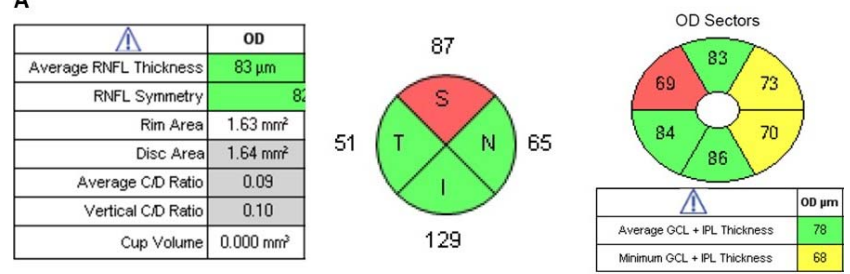

B
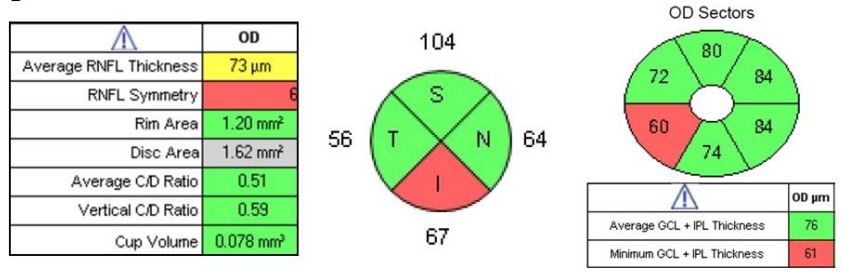

Figure 4. Optical coherence tomography images of two cases of suspected early glaucoma (right eyes) with normal visual fields. (A) Left: Average RNFL and optic disc parameters with values within normal limits. Middle: RNFL quadrants with superior quadrant out of normal limits (red). Right: GCIPL areas with temporal superior area out of normal limits (red) and borderline nasal areas (yellow). The minimum GCIPL is borderline. In this case, the glaucoma diagnostic calculator value was $2.7 \%$, indicating a low predictive probability of glaucoma. (B) Bottom left: Borderline average RNFL (yellow) and within normal limits of optic disc parameters (green). The disc area was $1.62 \mathrm{~mm}^{2}$, which is similar to that in case A. Middle: RNFL quadrants with inferior quadrant out of normal limits (red). Right: GCIPL areas with temporal inferior area out of normal limits (red). The minimum GCIPL is red. In this case, the glaucoma diagnostic calculator value was $75.2 \%$, suggesting a high probability of glaucoma.

formula may also assist the diagnosis of early glaucoma in cases with artifacts in the qualitative OCT analysis (Fig. 4), and it provides a new parameter that can facilitate, in addition to other tests, the glaucoma diagnosis in the clinical practice.

In conclusion, the GCIPL and RNFL parameters had similar diagnostic value, while the combined function increased the diagnostic value of these single parameters. The combined model includes information from 8 parameters from three structures, ONH, peripapillary RNFL, and GCIPLs, and requires only 1 minute to determine the probability of glaucoma in a suspected patient using the diagnostic calculator. The function is applicable even in large and small optic discs that do not show color classification in the OCT report (gray). In light of these results, the use of predictive models using a combination of parameters from Cirrus OCT improves glaucoma detection. The availability of the multivariate function allows external validation in other datasets (from different racial and geographic origins) and its potential use in clinical practice as another tool to interpret the OCT data analysis.

\section{Acknowledgments}

Supported in part by Instituto de Salud Carlos III, Red temática de Investigación Cooperativa, Proyecto RD07/0063: Patología ocular del envejecimiento, calidad visual y calidad de vida. The authors alone are responsible for the content and writing of the paper.

Disclosure: J.M. Larrosa, None; J. Moreno-Montañés, None; J.M. Martinez-de-la-Casa, None; V. Polo, None; Á. VelázquezVilloria, None; C. Berrozpe, None; M. García-Granero, None

\section{References}

1. Quigley HA. Number of people with glaucoma worldwide. $\mathrm{Br}$ J Ophthalmol. 1996;80:389-393. 
2. Azuara-Blanco A, Katz LJ, Spaeth GL, et al. Clinical agreement among glaucoma experts in the detection of glaucomatous changes of the optic disk using simultaneous stereoscopic photographs. Am J Ophthalmol. 2003;136:949-950.

3. Tan O, Chopra V, Lu AT, et al. Detection of macular ganglion cell loss in glaucoma by Fourier-domain optical coherence tomography. Opbthalmology. 2009;116:2305-2314.

4. Kim NR, Lee ES, Seong GJ, et al. Structure-function relationship and diagnostic value of macular ganglion cell complex measurement using Fourier-domain OCT in glaucoma. Invest Ophthalmol Vis Sci. 2010;51:4646-4651.

5. Mwanza JC, Durbin MK, Budenz DL, et al. Glaucoma diagnostic accuracy of ganglion cell-inner plexiform layer thickness: comparison with nerve fiber layer and optic nerve head. Ophthalmology. 2012;119:1151-1158.

6. Huang JY, Pekmezci M, Mesiwala N, Kao A, Lin S. Diagnostic power of optic disc morphology, peripapillary retinal nerve fiber layer thickness, and macular inner retinal layer thickness in glaucoma diagnosis with Fourier-domain optical coherence tomography. J Glaucoma. 2011;20:87-94.

7. Fang Y, Pan YZ, Li M, Qiao RH, Cai Y. Diagnostic capability of Fourier-domain optical coherence tomography in early primary open angle glaucoma. Chin Med J (Engl). 2010;123:20452050.

8. Mwanza JC, Warren JL, Budenz DL, et al. Combining spectral domain optical coherence tomography structural parameters for the diagnosis of glaucoma with early visual field loss. Invest Ophthalmol Vis Sci. 2013;54:8393-8400.

9. Mills RP, Budenz DL, Lee PP, et al. Categorizing the stage of glaucoma from pre-diagnosis to end-stage disease. Am J Ophthalmol. 2006;141:24-30.
10. Hanley JA, McNeil BJ. A method of comparing the areas under receiver operating characteristic curves derived from the same cases. Radiology. 1983;148:839-843.

11. Efron B, Tibshirani RJ. An Introduction to the Bootstrap. London, UK: Chapman \& Hall; 1993.

12. Brunelli A, Rocco G. Internal validation of risk models in lung resection surgery: bootstrap versus training-and-test sampling. J Thorac Cardiovasc Surg. 2006;131:1243-1247.

13. Medeiros FA, Zangwill LM, Bowd C, Vessani RM, Susanna R Jr, Weinreb RN. Evaluation of retinal nerve fiber layer, optic nerve head, and macular thickness measurements for glaucoma detection using optical coherence tomography. Am J Ophthalmol. 2005;139:44-55.

14. Leung CK, Chan WM, Yung WH, et al. Comparison of macular and peripapillary measurements for the detection of glaucoma: an optical coherence tomography study. Ophthalmology. 2005;112:391-400.

15. Wollstein G, Schuman JS, Price LL, et al. Optical coherence tomography (OCT) macular and peripapillary retinal nerve fiber layer measurements and automated visual fields. $A m \mathrm{~J}$ Ophthalmol. 2004;138:218-225.

16. Leal-Fonseca M, Rebolleda G, Oblanca N, Moreno-Montañes J, Muñoz-Negrete J. A comparison of false positives in retinal nerve fiber layer, optic nerve head and macular ganglion cellinner plexiform layer from two spectral-domain optical coherence tomography devices. Graefes Arch Clin Exp Ophthalmol. 2014;252:321-330.

17. Baskaran M, Ong EL, Li JL, et al. Classification algorithms enhance the discrimination of glaucoma from normal eyes using high-definition optical coherence tomography. Invest Ophthalmol Vis Sci. 2012;53:2314-2320. 